\title{
THE SEMIGROUP OF ONE-TO-ONE TRANSFORMATIONS WITH FINITE DEFECTS
}

\author{
by INESSA LEVI and BORIS M. SCHEIN
}

(Received 25 March, 1988)

Let $\mathscr{S}$ be the semigroup of all total one-to-one transformations of an infinite set $X$. For an $f \in \mathscr{S}$ let the defect of $f$, def $f$, be the cardinality of $X-R(f)$, where $R(f)=f(X)$ is the range of $f$. Then $\mathscr{S}$ is a disjoint union of the symmetric group $\mathscr{G}_{X}$ on $X$, the semigroup $S$ of all transformations in $\mathscr{S}$ with finite non-zero defects and the semigroup $\bar{S}$ of all transformations in $S$ with infinite defects, such that $S \cup \bar{S}$ and $\bar{S}$ are ideals of $\mathscr{S}$. The properties of $\mathscr{G}_{X}$ and $\bar{S}$ have been investigated by a number of authors (for the latter it was done via Baer-Levi semigroups, see [2], [3], [5], [6], [7], [8], [9], [10] and note that $\bar{S}$ decomposes into a union of Baer-Levi semigroups). Our aim here is to study the semigroup $S$. It is not difficult to see that $S$ is left cancellative (we compose functions $f, g$ in $S$ as $f g(x)=f(g(x))$, for $x \in X)$ and idempotent-free. All automorphisms of $S$ are inner [4], that is of the form $f \mapsto h f h^{-1}, f \in S, h \in \mathscr{G}_{X}$.

In the present paper, we are concerned with congruences, Green's relations and ideals of $S$. A large variety of distinct types of congruences on $S$ is present and the main results are the content of Theorems 4,5 and 6 . In the concluding remark we state some unsolved problems and conjectures on congruences on $S$.

For $f, g \in S$, let $D(f, g)=\{x: f(x) \neq g(x)\}$. The next lemma is easily verified.

Lemma 1. Let $f, g, t$ be one-to-one transformations. Then

(i) $\operatorname{def}(f g)=\operatorname{def} f+\operatorname{def} g$;

(ii) $D(t f, t g)=D(f, g)$;

(iii) $t(D(f t, g t))=D(f, g) \cap R(t)$.

Let $\mathscr{L}, \mathscr{R}, \mathscr{H}, \mathscr{D}, \mathscr{J}$ be the Green's relations on $S[1$, p. 47-49] and $i$ be the diagonal congruence. For an $f \in S$ let $R_{f}\left[L_{f}, J_{f}\right]$ denote the principal right [left, two-sided] ideal of $S$ generated by $f$. Denote by $\mathbb{N}$ the set of all natural numbers. Given $n \in \mathbb{N}$ let

$$
C_{n}=\{f \in S: \operatorname{def} f=n\}, I_{n}=\bigcup\left\{C_{k}: k \geq n\right\} .
$$

It follows from Lemma 1 that for every $n \in \mathbb{N}, I_{n}$ is an ideal of $S$.

Proposition 2.(i) $\mathscr{L}=\mathscr{R}=\mathscr{H}=\mathscr{D}=\mathscr{J}=i$ on $S$. For every $f \in S$,

(ii) $L_{f}=J_{f}=\{f\} \cup I_{n}$, where $n=1+\operatorname{def} f$;

(iii) $R_{f}=f \cup T(A)$, where $A=R(f)$ and $T(A)=\{g \in S: R(g) \varsubsetneqq A\}$.

(iv) $A$ subset $I$ of $S$ is an ideal of $S$ if and only if $I=B \cup I_{n}$ for some $n \geq 2$ and $B \subseteq C_{n-1}$.

Proof. (i)-(iii) can be easily verified using Lemma 1, while (iv) follows from (ii) and an observation that every ideal is a union of principal ideals.

We remark that not every ideal of $S$ is principal, for example, if $B$ is a proper subset

Glasgow Math. J. 31 (1989) 243-249. 
of $C_{1}$ consisting of more than one element, then the ideal $B \cup I_{2}$ is a non-principal ideal of $S$.

For an ideal $I$ of a semigroup $S$ the Rees ideal congruence $I^{*}$ is such that $(f, g) \in I^{*}$ iff either $f=g$ or $f, g \in I$, where $f, g \in S$. As usual, we write $S / I$ for $S / I^{*}$. Observe that Proposition 2 (iv) describes all Rees ideal congruences on $S$.

Another type of congruences on $S$ is defined as follows. Let $\alpha$ be an infinite cardinal that does not exceed $|X|^{+}$, the cardinal successor of $|X|$. Then a relation $\Delta_{\alpha}$ on $S$ for which $(f, g) \in \Delta_{\alpha}$ iff $|D(f, g)|<\alpha$ is a congruence on $S$.

Let $\delta$ be a relation on $S$ such that for $f, g \in S,(f, g) \in \delta$ iff $\operatorname{def} f=\operatorname{def} g$. Lemma 1 ensures that $\delta$ is a congruence on $S$.

PROPOSITION 3. (i) For every infinite $\alpha \leq|X|^{+}, \Delta_{\alpha}$ is a cancellative congruence on $S$.

(ii) $\Delta_{\aleph_{0}} \subseteq \delta$.

Proof. While (i) follows from Lemma 1, to prove (ii) let $(f, g) \in \Delta_{x_{0}}, D(f, g)=D$, $f(D)=A, g(D)=B$, and $(X-R(f)) \cap(X-R(g))=C$. Then $X-R(f)=[(X-R(f)) \cap$ $R(g)] \cup[(X-R(f)) \cap(X-R(g))]=[(X-R(f)) \cap B] \cup C=(B-A) \cup C . \quad$ Similarly, $X-R(g)=(A-B) \cup C$, and the result follows from the fact that $|A|=|D|=|B|<\aleph_{0}$.

Now we are in a position to present our main result on congruences on $S$. These are given in Theorems 4,5 and 6 below. Theorem 4 describes all the congruences $\lambda \subseteq \Delta_{\aleph_{0}}$. It is shown that for every such $\lambda$ there exists $n \in \mathbb{N}$ such that $\lambda$ coincides with $\Delta_{\varkappa_{0}}$ on $I_{n}$. Our description of $\lambda \subseteq \Delta_{\varkappa_{0}}$ is given in terms of equivalence series defined below.

Let $\rho_{k}$ be an equivalence on $C_{k}, k \geq 1$. We say that an equivalence $\rho_{l}$ on $C_{l}, l>k$, is derived from $\rho_{k}$ if whenever $(f, g) \in \rho_{k}, t \in S$ with def $t=l-k$ then $(f t, g t),(t f, t g) \in \rho_{l}$. Every congruence $\lambda$ on $S$ induces in a natural way an equivalence $\bar{\lambda}$ on $C_{k}, k \geq 1$. Given an equivalence $\rho_{k}$ on $C_{k}$ such that $\rho_{k} \subseteq \tilde{\Delta}_{\boldsymbol{x}_{0}}$ let $\Sigma_{k}$ denote a set of equivalences $\rho_{l}$ on $C_{l}$, $l \geq k$, such that for every $l>k, \rho_{l} \varsubsetneqq \tilde{\Delta}_{\aleph_{0}}$ and $\rho_{l}$ is derived from $\rho_{l-1}$. We refer to such $\Sigma_{k}$ as an equivalence series derived from $\rho_{k}$. We show (Lemma 10) that every such series is finite and hence we can define a maximal equivalence series $\Sigma_{k}$ derived from $\rho_{k}$ such that if $m=\max \left\{l: \rho_{l} \in \Sigma_{k}\right\}$ then every equivalence derived from $\rho_{m}$ coincides with $\Delta_{\aleph_{0}}$.

THEOREM 4. Let $n \in \mathbb{N}^{-}, \rho_{n} \subseteq \tilde{\Delta}_{\aleph_{0}}$ be a non-trivial equivalence on $C_{n}$ and $\Sigma_{n}$ be an equivalence series derived from $\rho_{n}$ with $m=\max \left\{l: \rho_{l} \in \Sigma_{n}\right\}$. Then

$$
\rho=i \cup\left\{\rho_{k}: \rho_{k} \in \Sigma_{n}\right\} \cup\left(\Delta_{\aleph_{0}} \cap\left(I_{m+1} \times I_{m+1}\right)\right)
$$

is a congruence on $S$ contained in $\Delta_{\alpha_{0}}$.

Conversely, if $\lambda \subseteq \Delta_{\varkappa_{0}}$ is a non-diagonal congruence on $S$ then there exists a non-trivial equivalence $\rho_{n} \subseteq \bar{\Delta}_{\aleph_{0}}$ on $C_{n}$ and a maximal equivalence series $\Sigma_{n}$ derived from $\rho_{n}$ such that $\lambda=\rho$ as defined above.

In the following we describe congruences $\Delta_{\alpha}, \aleph_{0} \leq \alpha \leq|X|^{+}$. We observe that every $\Delta_{\alpha}$ can be extended in a natural way to a congruence $\Delta_{\alpha}^{\prime}$ on $\mathscr{G}_{X} \cup S$, (so that for $f$, $g \in \mathscr{G}_{X} \cup S,(f, g) \in \Delta_{\alpha}$ if $\left.|D(f, g)|<\alpha\right)$. Note that $\Delta_{|X|^{+}}=S \times S$, the universal congruence on $S$. Let $\Lambda$ be the lattice of congruences on $S$, and if $\rho, \sigma \in \Lambda$, write

$$
[\rho, \sigma]=\{\gamma \in \Lambda: \rho \subseteq \gamma \subseteq \sigma\},[\rho)=\{\gamma \in \Lambda: \rho \subseteq \gamma\},(\rho)=\{\gamma \in \Lambda: \rho \sqsubseteq \gamma\}
$$


THEOREM 5. (i) $\Delta_{\varkappa_{0}}$ is a minimal cancellative idempotent-free congruence on $S$.

For every $\kappa_{0}<\alpha \leq|X|^{+}$the following holds:

(ii) $\Delta_{\alpha}$ is a group congruence on $S$ and $\left(\mathscr{G}_{X} \cup S\right) / \Delta_{\alpha}^{\prime} \cong S / \Delta_{\alpha} \cong \mathscr{G}_{X} / \Delta_{\alpha}^{\prime}$;

(iii) $\left[\Delta_{\alpha}\right)=\left\{\Delta_{\beta}: \beta>\alpha\right\}$.

For $m, n \in \mathbb{N}$, let $\sigma(m, n)$ be a congruence on $S$ such that $(f, g) \in \sigma(m, n)$ if either $f=g$ or $\operatorname{def} f, \operatorname{def} g \geq m$ and $\operatorname{def} f \equiv \operatorname{def} g \bmod n$. Evidently $\delta \subseteq \sigma(m, n)$. Moreover, if a congruence $\gamma$ contains $\delta$, then $S / \gamma$ is isomorphic to a homomorphic image of $S / \delta \cong(\mathbb{N},+)$. Hence $S / \gamma$ is isomorphic to a monogenic subsemigroup of type $(m, n)$, for some $m, n \in \mathbb{N}$. It follows that $\gamma=\sigma(m, n)$. This proves the first part of Theorem 6 below. Now let $\mathbb{N}_{1}$ be the lattice of natural numbers ordered by divisibility, $\mathbb{N}_{1}^{*}$ be the dual lattice. Let $\omega$ be the first infinite ordinal, $\omega^{*}$ be the dual order type. For a lattice $A$ let $A^{0}$ denote the lattice obtained from $A$ by adjoining a (new) least element 0 .

THEOREM 6. (i) $[\delta)=\{\sigma(m, n): m, n \in \mathbb{N}\}$.

(ii) $[\delta) \cong\left(\omega^{*} \times \mathbb{N}_{1}^{*}\right)^{0}\left(\cong\left(\left(\omega \times \mathbb{N}_{1}\right)^{*}\right)^{0}\right)$.

(iii) For every $n>1, \sigma(1, n)$ is a group congruence on $S$ and $S / \sigma(1, n) \cong Z_{n}$, a cyclic group of order $n$.

(iv) There is no least group congruence on $S$.

For every $\alpha>\kappa_{0}$,

(v) $S / \delta \cap \Delta_{\alpha} \cong\left(\mathscr{G}_{X} / \Delta_{\alpha}^{\prime}\right) \times(\mathbb{N},+)$;

(vi) $S / \sigma(m, n) \cap \Delta_{\alpha} \cong\left(\mathscr{G}_{X} / \Delta_{\alpha}^{\prime}\right) \times(\mathbb{N},+) / \eta(m, n)$,

where $\eta(m, n)$ is a congruence on the semigroup $(\mathbb{N},+)$ such that $(k, l) \in \eta(m, n)$ if either $k=l$ or $k, l \geq m$ and $k \equiv l \bmod n$;

(vii) for $m, n \in \mathbb{N}, \mathscr{A} \subseteq C_{m} / \Delta_{\alpha}, \mathscr{A} \neq \varnothing, A=\bigcup \mathscr{A}$, and $\bar{A}=\left(\bigcup_{i=1}^{m} C_{i}\right)-A$

$\theta(\alpha, m, n, \mathscr{A})=\left(\delta \cap \Delta_{\alpha} \cap(\bar{A} \times \bar{A})\right) \cup\left(\sigma(m, n) \cap \Delta_{\alpha} \cap\left(\left(I_{m+1} \cup A\right) \times\left(I_{m+1} \cup A\right)\right)\right.$

is a congruence on $S$.

(viii) every congruence $\gamma \in\left(\delta \cap \Delta_{\alpha}, \Delta_{\alpha}\right), \gamma \nsubseteq \delta$, has the form $\theta(\alpha, m, n, \mathscr{A})$ as described in (vii).

Proofs of Theorems 4, 5 and 6 constitute the remainder of the paper.

Lemma 7. Given $f, g, t, s \in S$ such that $D(f, g)=D(t, s)=\{x\}$, where $x \in X$, and $\operatorname{def} f=\operatorname{def} g>\operatorname{def} s=\operatorname{def} t$, there exists $l \in S$ with $l s=f$ and $l t=g$.

Proof. Observe that $g(x) \notin R(f), t(x) \notin R(s)$ and choose a 1-1 function

$$
l_{1}: X-R(s)-\{t(x)\} \rightarrow X-R(f)-\{g(x)\},
$$

(note that since $\operatorname{def} s<\operatorname{def} f, l_{1}$ has a finite non-zero defect in $X-R(f)-\{g(x)\}$ ). Define a function $l: X \rightarrow X$ as follows. For a $y \in X$, let

$$
l(y)=\left\{\begin{array}{l}
f(z) \text { if } y=s(z) \quad \text { for some } z \in X, \\
g(x) \text { if } y=t(x), \\
l_{1}(y) \text { if } y \in X-R(s)-\{t(x)\} .
\end{array}\right.
$$

Clearly, $l \in S$ and $l s=f$, also $l t(x)=g(x)$ and for $u \neq x, l t(u)=l s(u)=f(u)$, so that $l t=f$. 
Lemma 8. Let $(f, g) \in \Delta_{\chi_{0}}, f \neq g$. Then there exist $f_{1}, \ldots, f_{n} \in S$ such that $\left|D\left(f_{i}, f_{i+1}\right)\right|=$ 1 , for $i=1, \ldots, n-1$, and $f_{1}=f, f_{n}=g$.

Proof. Let $|D(f, g)|=m$. We prove the result by induction on $m$. Assume that the statement is true for every pair of functions in $S$ that differ on at most $m-1$ points. Let $D(f, g)=D, f(D)=A$ and $g(D)=B$. Consider two cases:

(i) $A \neq B$.

Choose $b \in B-A$, and let $g(d)=b$, where $d \in D$. Let $f_{1}=f$ and define $f_{2}$ as follows: $\left.f_{2}\right|_{X-\{d\}}=\left.f_{1}\right|_{X-\{d\}}$ and $f_{2}(d)=b$. Then $D\left(f_{1}, f_{2}\right)=\{d\}$ and $D\left(f_{2}, g\right)=D-\{d\}$. By induction supposition there are $f_{3}, \ldots, f_{n} \in S$ such that $f_{n}=g$ and $\left|D\left(f_{i}, f_{i+1}\right)\right|=1$ for every $i=2,3, \ldots, n-1$. Thus $f_{1}, f_{2}, f_{3}, \ldots, f_{n}$ is the required sequence.

(ii) $A=B$.

Using an observation that $A=B$ implies that $X-(R(f) \cup R(g)) \neq \varnothing$, choose $x \in$ $X-(R(f) \cup R(g))$. Choose $d \in D$, let $f_{1}=f$ and define $f_{2}$ so that $\left.f_{2}\right|_{X-\{d\}}=\left.f_{1}\right|_{X-\{d\}}$ and $f_{2}(d)=x$. Let $g_{1}$ be such that $\left.g_{1}\right|_{X-\{d\}}=\left.g\right|_{X-\{d\}}$ and $g_{1}(d)=x$.

Then $D\left(f_{1}, f_{2}\right)=D\left(g_{1}, g\right)=\{d\}$ and $D\left(f_{2}, g_{1}\right)=D-\{d\}$, so there exist $f_{3}, \ldots, f_{n} \in S$ with $f_{n}=g_{1}$ and $\left|D\left(f_{i}, f_{i+1}\right)\right|=1$ for every $i=2, \ldots, n-1$. The result follows.

LemMA 9. Let $\lambda$ be a congruence on $S, \lambda \subseteq \Delta_{x_{0}}$. Let

$N=\{\operatorname{def} f: f \in S,|[f]| \neq 1,[f] \in S / \lambda\}$, and for every $n \in N$ let

$L(n)=\{|D(f, g)|: f, g \in S, f \neq g,(f, g) \in \lambda, \operatorname{def} f=n\}$,

$\Psi_{n}: L(n) \rightarrow \mathbb{N}$ be defined by $\Psi_{n}(l)=n+l+1, l \in L(n)$. Let $m_{n}$ be a minimum value of $\Psi_{n}$ and

$$
m=\min \left\{m_{n}: n \in N\right\} .
$$

Then $\lambda \cap\left(I_{m} \times I_{m}\right)=\Delta_{\aleph_{0}} \cap\left(I_{m} \times I_{m}\right)$.

Proof. Let $m=m_{n}=n+l+1$, for some $n \in N$ and $l \in L(n)$. Let $f, g \in S$ such that $(f, g) \in \lambda, f \neq g, \operatorname{def} f=\operatorname{def} g=n$ and $|D(f, g)|=l$. Let $(t, s) \in \Delta_{\varkappa_{0}}$ with $\operatorname{def} t=\operatorname{def} s=k \geq$ $m$. In view of Lemma 8 we can assume that $|D(t, s)|=1$. Let $D(t, s)=\{x\}, D(f, g)=D$. Choose $d \in D, a \in X-D$ and $q \in S$ with $R(q)=(X-D) \cup\{d\}-\{a\}$ and $q(x)=d$. Then $D(f q, g q)=\{x\}$, def $f q=\operatorname{def} g q=n+|D|=m-1<k$, and so Lemma 7 implies there exists $l \in S$ such that $l f q=t, \lg q=s$, so that $(t, s) \in \lambda$.

LEMMA 10. Every equivalence series $\Sigma_{k}$ derived from $\rho_{k} \subseteq \bar{\Delta}_{\aleph_{0}}$ is finite.

Proof. The result follows from the proof of Lemma 9 and an observation that the relation derived from is transitive, that is if $l_{i} \in \mathbb{N}, i=1,2,3$ and $\rho_{l_{i}}$ is an equivalence on $C_{l_{i}}$ such that $\rho_{l_{i+1}}$ is derived from $\rho_{l_{i}}, i=1,2$, then $\rho_{l_{3}}$ is derived from $\rho_{l_{1}}$.

Proof of Theorem 4. That $\rho$ defined in (1) is a congruence on $S$ follows from the definition of a congruence series derived from $\rho_{n}$ and Lemma 10.

To show the converse let $\lambda<\Delta_{x_{0}}$ be a non-diagonal congruence on $S$ and let $n$ be the minimal integer such that $\lambda \cap\left(C_{n} \times C_{n}\right) \neq i \cap\left(C_{n} \times C_{n}\right), m$ be the maximal integer with 
$\lambda \cap\left(C_{m} \times C_{m}\right) \neq \Delta_{\aleph_{0}} \cap\left(C_{m} \times C_{m}\right) \quad$ if $\quad \lambda \cap\left(C_{n} \times C_{n}\right) \neq \Delta_{\aleph_{0}} \cap\left(C_{n} \times C_{n}\right) \quad$ and $m=n$ otherwise. Then for every $k, n \leq k \leq m, \lambda$ induces in a natural way equivalences $\rho_{k}$ on $C_{k}$. The set of all these equivalences forms a maximal congruence series $\Sigma_{n}$ derived from $\rho_{n}$ and the result follows.

COROLLARY 11. (i) Every right [left] cancellative congruence $\eta$ on $S$ contains $\Delta_{\mathrm{x}_{0}}$.

(ii) Every group congruence on $S$ contains $\Delta_{\aleph_{0}}$.

Proof. (i) Assume $\eta \supsetneqq \Delta_{\aleph_{0}}$ is right cancellative, and let $(f, g) \in \Delta_{\varkappa_{0}}$. Let $m$ be such that $\left(\Delta_{\aleph_{0}} \cap \rho\right) \cap\left(I_{m} \times I_{m}\right)=\Delta_{\varkappa_{0}} \cap\left(I_{m} \times I_{m}\right)$ (Lemma 9) and $t \in C_{m}$. Then (ft, gt) $\in \Delta_{x_{0}} \cap \eta$, so that $(f, g) \in \eta$ since $\eta$ is right cancellative.

(ii) Follows from (i).

The next lemma is self-evident.

Lemma 12. For any $f \in \mathscr{G}_{X} \cup S, n \geq 0$ there exists $g \in \mathscr{G}_{X} \cup S$ with $\operatorname{def} g=n$ and $(f, g) \in \Delta_{\kappa_{0}}^{\prime}$, where $\aleph_{1}=\aleph_{0}^{+}$.

Proof of Theorem 5. (i) It is not difficult to verify that $\Delta_{\varkappa_{0}}$ is a cancellative congruence. Hence, in view of Corollary 11 (i) it is sufficient to show that $\Delta_{\varkappa_{0}}$ is idempotent-free. This follows from an observation that for any $f \in S, D\left(f, f^{2}\right)=$ $D\left(f i_{X}, f^{2}\right)=D\left(i_{X}, f\right)$ (where $i_{X}$ is the identity mapping on $X$ ), so that $\left|D\left(f, f^{2}\right)\right| \geq \aleph_{0}$ and $\left(f, f^{2}\right) \notin \Delta_{x_{0}}$.

(ii) It is sufficient to show the existence of the indicated isomorphisms. For that let $f \in S, h \in \mathscr{G}_{X},[f]$ and $[h]$ be $\Delta_{\alpha}^{\prime}$-classes of $f$ in $S$ and $h$ in $\mathscr{G}_{X}$ respectively. We show that $[f] \cup[h]$ is the $\Delta_{\alpha}^{\prime}$-class of $f$ (or, equivalently, $\left.h\right)$ in $\mathscr{G}_{X} \cup S$ if any only if $(f, h) \in \Delta_{\alpha}^{\prime}$. While the necessity is clear the sufficiently follows from the observation that if $(f, h) \in \Delta_{\alpha}^{\prime}$ and $A$ is the $\Delta_{\alpha}^{\prime}$ class of $f$ in $\mathscr{G}_{X} \cup S$ then $A \cap \mathscr{G}_{X}=[h]$ and $A \cap S=[f]$.

(iii) Let $\gamma$ be a congruence on $S$ containing $\Delta_{\alpha}$. Since $S / \Delta_{\alpha} \cong \mathscr{G}_{X} / \Delta_{\alpha}$ there exists a homomorphism from $\mathscr{G}_{X} / \Delta_{\alpha}^{\prime}$ onto $S / \gamma$, so that there is a congruence $\gamma^{\prime}$ on $\mathscr{G}_{X}$ such that $\mathscr{G}_{X} / \gamma^{\prime} \cong S / \gamma$. But then $\gamma^{\prime}=\Delta_{\beta}$ for some $\beta \geq \alpha$ and so

$$
S / \gamma \cong \mathscr{G}_{X} / \Delta_{\beta}^{\prime} \cong S / \Delta_{\beta}, \quad \text { and } \quad \gamma=\Delta_{\beta} .
$$

The next result describes some properties of the congruence $\delta$. Recall that $(f, g) \in \delta$ if $\operatorname{def} f=\operatorname{def} g$.

Proposition 13. (i) $\delta \vee \Delta_{\mathrm{x}_{1}}=S \times S$.

(ii) $\delta$ is the unique congruence on $S$ such that $S / \delta \cong(\mathbb{N},+)$, where $(\mathbb{N},+)$ denotes the semigroup of positive integers under addition.

Proof. (i) Let $f, g \in S$. According to Lemma 12, there exists $k \in S$ such that $(f, k) \in \delta,(k, g) \Delta_{x_{1}}$, so that $(f, g) \in \Delta_{x_{1}} \circ \delta \subseteq \delta \vee \Delta_{x_{1}}$.

(ii) We show that if $\theta$ is a homomorphism from $S$ onto $(\mathbb{N},+)$ then $\theta^{-1} \circ \theta=\delta$. Firstly observe that if $f \in C_{m}$ and $g \in C_{n}$ with $m<n$, then $\theta(f)<\theta(g)$, for there exists $q \in S$ such that $q f=g$ and $\theta(q)+\theta(f)=\theta(g)$. Since $\theta$ is onto, $\theta\left(C_{1}\right)=1$ and so $\theta\left(C_{m}\right)=m$, for every $m \in \mathbb{N}$ (indeed, if $f \in C_{m}$ then there exist $f_{1}, f_{2}, \ldots, f_{m} \in C_{1}$ such that $f=f_{1} f_{2} \ldots f_{m}$, so that $\left.\theta(f)=\theta\left(f_{1}\right)+\theta\left(f_{2}\right)+\ldots+\theta\left(f_{m}\right)=m\right)$. 
Proof of Theorem 6. Let $\sigma(m, n) \rightarrow(m, n)$ be a mapping of $(\delta)$ onto $\mathbb{N} \times \mathbb{N}$. The result then follows from an observation that $\sigma\left(m_{1}, n_{1}\right) \subseteq\left(m_{2}, n_{2}\right)$ if and only if $m_{2} \leq m_{1}$ and $n_{2}$ divides $n_{1}$.

(iii) Follows from the fact that a monogenic semigroup of type $(m, n)$ is a group if $m=0$ and Proposition 13.

(iv) Observe firstly that $\bigcap\{\sigma(1, n): n \in \mathbb{N}\}=\delta$, indeed, if $(f, g) \in \bigcap\{\sigma(1, n): n \in$ $\mathbb{N}\}$, then $\operatorname{def} f \equiv \operatorname{def} g \bmod n$ for every $n \in \mathbb{N}$, so that $\operatorname{def} f=\operatorname{def} g$, or $(f, g) \in \delta$. If there is the least group congruence $\tau$ on $S$, then $\tau \subseteq \sigma(1, n)$ for every $n$, so that $\tau \subseteq$ $\cap\{\sigma(1, n): n \in \mathbb{N}\}=\delta$. It follows that $\delta$ is a group congruence (for $S / \delta \cong(S / \tau) /(\delta / \tau)$, which is a homomorphic image of the group $S / \tau$, a contradiction to Proposition 13).

The verification of (v)-(vii) is trivial.

(viii) Let $\gamma \in\left(\delta \cap \Delta_{\alpha}, \Delta_{\alpha}\right), \gamma \notin \delta, m$ be the minimal integer for which there exists $f \in S$ with def $=m$ such that the $\gamma$-class of $f$ does not coincide with the $\left(\delta \cap \Delta_{\alpha}\right)$-class of $f$. Let $\mathscr{B}$ be the set of all classes of $\gamma$ that contain an element $g$ of $C_{m}$ and that do not coincide with the $g$-class of $\delta \cap \Delta_{\alpha}$ and $\mathscr{A}=\left\{C_{m} \cap B: B \in \mathscr{B}\right\}$. Let $k=\min \{\operatorname{def} g:(f, g) \in$ $\gamma,(f, g) \notin \delta\}$ and $n=k-m$. Clearly, $\gamma$ coincides with $\delta \cap \Delta_{\alpha}$ on $\bigcup\left\{C_{l}: 1 \leq l \leq m-1\right\}$. We show firstly that

$$
\gamma \cap\left(I_{m+1} \times I_{m+1}\right) \supseteq \sigma(m, n) \cap \Delta_{\alpha} \cap\left(I_{m+1} \times I_{m+1}\right) .
$$

For $f \in S$ let $S(f)=\{x \in X: f(x) \neq x\}$ and shift $f=|S(f)|$.

Let $(f, g) \in \gamma$ with $\operatorname{def} f=m$, $\operatorname{def} g=m+n$. We observe that for every $t \in C_{1}$ with shift $t=\aleph_{0},\left(t^{n} f, g\right) \in \delta \cap \Delta_{\alpha} \subseteq \gamma$, so that $\left(f, t^{n} f\right) \in \gamma$. Moreover, for any integer $l>0$,

$$
\left(f, t^{\ln } f\right) \in \gamma,
$$

since $\left(f, t^{n} f\right) \in \gamma$ implies $\left(t^{n} f, t^{2 n} f\right) \in \gamma$, so that $\left(f, t^{2 n} f\right) \in \gamma$ etc.

Take $(p, q) \in \sigma(m, n) \cap \Delta_{\alpha} \cap\left(I_{m+1} \times I_{m+1}\right)$, and let $f$ be as above. Since def $p>m=$ def $f$ there exists an $s$ in $S$ such that $p=s f$. Without loss of generality assume that $\operatorname{def} q$-def $p=a n, a \in \mathbb{N}, a>0$. Now (3) implies that $\left(s f, s t^{a n} f\right) \in \gamma$, or $\left(p, s t^{a n} f\right) \in \gamma$. Also, $\operatorname{def}\left(s t^{a n} f\right)=\operatorname{def} q$ and $D\left(s t^{a n} f, q\right) \subseteq D(p, q) \cup f^{-1}\left(S\left(t^{a n}\right)\right)$, so that $\left|D\left(s t^{a n} f, q\right)\right|<\alpha$ and $\left(s t^{a n} f, q\right) \in \delta \cap \Delta_{\alpha} \leq \gamma$. We conclude that $(p, q) \in \gamma$.

To complete the proof it suffices to show that if $(u, v) \in \gamma \cap\left(I_{m+1} \times I_{m+1}\right)$ then $(u, v) \in \sigma(m, n)$. Assume $m<\operatorname{def} u<\operatorname{def} v$ and let $(\operatorname{def} v)-(\operatorname{def} u)=b n+r$, where $b$, $r \in \mathbb{N}, 0 \leq r<n$. Let $f$ and $t$ be chosen as above. We show that if $r>0$ then $\left(f, t^{r} f\right) \in \gamma$, a contradiction to the choice of $n$ that assures that $r=0$ and so $(u, v) \in \sigma(m, n)$. Observe that there exists $w \in S$ and $l \in \mathbb{N}, l>0$ such that $t^{l n} f=w u$. But then $(w u, w v) \in \gamma$ implies that $\left(t^{l n} f, w v\right) \in \gamma$, so that using (3) we conclude that $(f, w v) \in \gamma$. Note that def $w v=$ $(l+b) n+m+r$ and since $\gamma \supseteq \delta \cap \Delta_{\alpha}$ we have $\left(f, t^{n(l+b)+r} f\right) \in \gamma$. By (3), we have that $\left(t^{r} f, t^{n(l+b)+r} f\right) \in \gamma$, so that $\left(f, t^{r} f\right) \in \gamma$.

REMARK. In this paper we described certain large classes of congruences and parts of the lattice of congruences on $S$. A description of all congruences and the lattice of congruences of $S$ is as yet an open problem. In particular, we conjecture that for every infinite cardinal $\alpha \leq|X|$, every congruence in the interval $\left(\Delta_{\alpha} \cap \delta, \Delta_{\alpha^{+}} \cap \delta\right)$ can be 
described in terms of a finite equivalence series derived from a given equivalence $\lambda$ on $C_{k}$ for some $k \geq 1$ such that $\bar{\Delta}_{\alpha} \leq \lambda \leq \bar{\Delta}_{\alpha^{+}}$as was done in Theorem 4 for congruences $\rho \subseteq \Delta_{\aleph_{0}}$.

\section{REFERENCES}

1. A. H. Clifford and G. B. Preston, Algebraic theory of semigroups, Math Surveys No. 7, Amer. Math. Soc., Providence, R.I., Vol. I (1961).

2. I. Levi, B. M. Schein, R. P. Sullivan and G. R. Wood, Automorphisms of Baer-Levi semigroups, J. London Math. Soc. (2) 28 (1983), 492-495.

3. I. Levi and G. R. Wood, On maximal subsemigroups of Baer-Levi semigroups, Semigroup Forum, 30 (1984), 99-102.

4. I. Levi, Automorphisms of normal transformation semigroups, Proc. Edinburgh Math. Soc., 28 (1985), 185-205.

5. D. Lindsey and B. Madison, The lattice of congruences on Baer-Levi semigroup, Semigroup Forum 12 (1976), 63-70.

6. B. W. Mielke, Regular congruences on Croisot-Teissier and Baer-Levi semigroups, $J$. Math. Soc. Japan 24 (1972), 539-551.

7. B. W. Mielke, Regular congruences on a simple semigroup with a minimal right ideal, Publ. Math. Debreceen 20 (1973), 79-84.

8. B. W. Mielke, Completely simple congruences on Croisot-Teissier semigroups, Semigroup Forum 9 (1975), 283-293.

9. B. M. Schein, Symmetric semigroups of one-to-one transformations, Second all-union symposium on the theory of semigroups, summaries of talks, Sverdlovsk (1979), 99 (Russian).

10. E. G. Shutov, Semigroups of one-to-one transformations, Dokl. Akad. Nauk. SSSR 140 (1961), 1026-1028.

Department of Mathematics

UNIVERSITY OF LOUISVILLE

LOUISVILLE,

KeNTUCKY 40292

Department of Mathematical Sciences

UNIVERSITY OF ARKANSAS

FAYETTEVILLE,

ARKANSAS 72701 